\title{
Capacity Bounds for a Class of Diamond Networks with Conferencing Relays
}

\author{
Wanyao Zhao, David Yiwei Ding, Ashish Khisti
}

\begin{abstract}
Diamond channels can model modern wireless communication infrastructure that use relays to increase network capacity. This letter investigates the effect of conferencing between the relays in a particular class of diamond networks with source-to-relay and conferencing channels modeled as noiseless bit-pipes of given capacities. A lower bound to the capacity for this network and an upper bound that is tighter than the cut-set bound are presented. Such network with specific multiple access channel (MAC) types are studied and the minimum conferencing link capacity needed to achieve full cooperation for each MAC is ascertained. It is found that such minimum conferencing link capacity for the Gaussian MAC must be equal to the difference between the full cooperation capacity and the backhaul capacity. However, such result does not hold in general as the binary adder MAC illustrates a counter-example.
\end{abstract}

Index Terms-Diamond channel, conferencing relays, Marton's coding, rate-splitting

\section{INTRODUCTION}

The rapid increase in the demand for wireless services, supported by the growth in mobile technology, saw the advancement of relay communication for coverage extension and capacity improvement in network infrastructures of modern and emerging wireless networks under the standard of WiMAX, LTE, 4G, and 5G. The diamond network is first studied by [1], and since has been used to model the communication between a source and a destination with the help of two relays. The diamond network is a cascade of two channels: the broadcast channel where the source communicates with two separate relays, and multiple access channel (MAC) where the two relays communicate with the destination. In [2], the authors derived a lower bound based on Marton's coding. A tighter upper bound than the cutset bound was found in [3] and [4]. In another work, MAC with conference was first studied by Willems in [5]. The conferencing links can allow for an increase in correlation between the two relay messages through, for example, exchanging each relay's message, thereby allowing the source to send independent messages to the relays without compromising the cooperative potential.

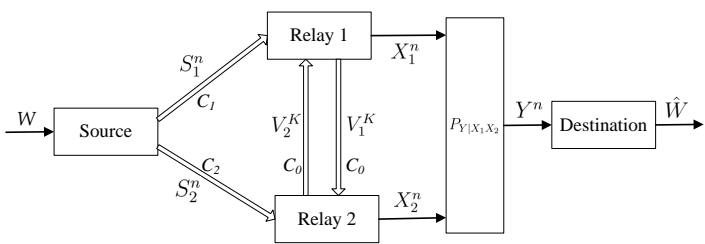

Fig. 1. Diamond network with relay conferencing.

In this letter, we combine the concepts of diamond network and MAC with conferencing to study the effect of relay conferencing on such networks. A key motivation for this study is that such model can represent many practical systems with relays that have out-of-band communication capacities, for example, nodes connected among each other via optical fiber or Wi-Fi. A particular subject of interest is to evaluate the impact of communication between the relays on the capacity bounds as compared to the diamond network without relay conferencing. For simplicity, it is assumed that the communication between the relays do not interfere with the communication of the message sent through the diamond network by treating the conferencing channels as noiseless bit-pipes as well. Such assumption is realistic in many practical systems where not only are the conferencing links out-of-band, but they are also wired (e.g. optical fiber), and therefore they do not interfere with the transmitted message.

\section{Problem SetuP}

We consider the diamond network in Fig. 11. The source node wishes to transmit message $W$ to the destination via two relays. The two relays can communicate via two conferencing links. The backhaul capacities for the noiseless bit-pipes connecting the source to relays 1 and 2 are $C_{1}$ and $C_{2}$, respectively. The capacity for both relay conferencing links is $C_{0}$.

The source encoder maps message $W \in\left[1: 2^{n R}\right]$ onto $S_{1}^{n}$ and $S_{2}^{n}$ and transmits through the noiseless bit-pipes to relays 1 and 2 , respectively, i.e.

$$
S_{1}^{n}=f_{0,1}(W), \quad S_{2}^{n}=f_{0,2}(W) .
$$

$S_{1}^{n}$ and $S_{2}^{n}$ are such that $H\left(S_{1}^{n}\right) \leq n C_{1}$ and $H\left(S_{2}^{n}\right) \leq$ $n C_{2}$. After receiving $S_{i}^{n}$, two relays can communicate 
through $K$ rounds in a round-robin fashion. At round $k$, relay $i$ sends $V_{i, k}$ to the other relay based on $S_{i}^{n}$ and message from the other relay in previous rounds, i.e.

$$
V_{1, k}=f_{1,2}\left(S_{1}^{n}, V_{2}^{k-1}\right), \quad V_{2, k}=f_{2,1}\left(S_{2}^{n}, V_{1}^{k-1}\right)
$$
for $k=1,2, \ldots, K$ and $V_{i, 0}=1$ for $i=1,2 . V_{i}^{K}$ satisfies the constraint $H\left(V_{i}^{K}\right) \leq n C_{0}$ for $i=1,2$. Then relay $i$ maps its received signal $S_{i}^{n}$ from the transmitter and $V_{\{1,2\} \backslash i}^{K}$ from the other relay to $X_{i}^{n}$ as the input to the MAC for $i=1,2$, i.e.

$$
X_{1}^{n}=f_{1,3}\left(S_{1}^{n}, V_{2}^{K}\right), \quad X_{2}^{n}=f_{2,3}\left(S_{2}^{n}, V_{1}^{K}\right) .
$$

The MAC is characterized by its input alphabet $\mathcal{X}_{1}, \mathcal{X}_{2}$, output alphabet $\mathcal{Y}$ and transition probability $p\left(y \mid x_{1}, x_{2}\right)$. The receiver decodes an estimate $\hat{W}=$ $g\left(Y^{n}\right)$ of $W$. The capacity of the diamond network considered is defined as the maximum $R$ such that for any $\epsilon>0$ there exists $\left\{f_{i, 0}, f_{i,\{1,2\} \backslash i}, f_{i, 3}, g\right\}, i=1,2$ and $n$ large enough such that $\operatorname{Pr}(W \neq \hat{W}) \leq \epsilon$.

\section{RESULTS ON BOUNDS}

Theorem 1. An upper bound of the diamond network with conference is

$$
R \leq \max _{p\left(x_{1}, x_{2}\right)} \min _{p\left(u \mid x_{1}, x_{2}, y\right)} \beta,
$$

where

$$
\beta=\min \left\{\begin{array}{l}
C_{1}+C_{2} \\
I\left(X_{1}, X_{2} ; Y\right) \\
C_{1}+C_{0}+I\left(X_{2} ; Y \mid X_{1}\right) \\
C_{2}+C_{0}+I\left(X_{1} ; Y \mid X_{2}\right) \\
\frac{1}{2}\left(C_{1}+C_{2}+2 C_{0}+I\left(X_{1}, X_{2} ; Y \mid U\right)\right. \\
\left.\quad+I\left(X_{1} ; U \mid X_{2}\right)+I\left(X_{2} ; U \mid X_{1}\right)\right) .
\end{array}\right.
$$

with $|\mathcal{U}| \leq\left|\mathcal{X}_{1}\right|\left|\mathcal{X}_{2}\right||\mathcal{Y}|+2$.

Proof: Starting from Fano's inequality, we have

$$
\begin{aligned}
n R & \leq I\left(W ; Y^{n}\right)+n \epsilon \\
& \leq I\left(S_{1}^{n}, S_{2}^{n} ; Y^{n}\right)+n \epsilon \\
& \leq H\left(S_{1}^{n}\right)+H\left(S_{2}^{n}\right)-I\left(S_{1}^{n} ; S_{2}^{n}\right)+n \epsilon \\
& \leq n C_{1}+n C_{2}-I\left(S_{1}^{n} ; S_{2}^{n}\right)+n \epsilon .
\end{aligned}
$$

Next we need to find the relation between $I\left(S_{1}^{n} ; S_{2}^{n}\right)$ and $I\left(X_{1}^{n} ; X_{2}^{n}\right)$, as follows,

$$
\begin{aligned}
& I\left(X_{1}^{n} ; X_{2}^{n}\right) \\
\leq & I\left(S_{1}^{n}, V_{2}^{K} ; S_{2}^{n}, V_{1}^{K}\right) \\
= & I\left(S_{1}^{n} ; S_{2}^{n}\right)+I\left(V_{2}^{K} ; S_{2}^{n} \mid S_{1}^{n}\right) \\
& +I\left(V_{1}^{K} ; S_{1}^{n} \mid S_{2}^{n}\right)+I\left(V_{1}^{K} ; V_{2}^{K} \mid S_{1}^{n}, S_{2}^{n}\right),
\end{aligned}
$$

where (11) is due to the Markov chain $X_{1}^{n} \leftrightarrow$ $\left(S_{1}^{n}, V_{2}^{K}\right) \leftrightarrow\left(S_{2}^{n}, V_{1}^{K}\right) \leftrightarrow X_{2}^{n}$. For the terms in (12),

$$
\begin{aligned}
I\left(V_{1}^{K} ; S_{1}^{n} \mid S_{2}^{n}\right) & =H\left(V_{1}^{K} \mid S_{2}^{n}\right) \\
I\left(V_{2}^{K} ; S_{2}^{n} \mid S_{1}^{n}\right) & =H\left(V_{2}^{K} \mid S_{1}^{n}\right) \\
I\left(V_{1}^{K} ; V_{2}^{K} \mid S_{1}^{n}, S_{2}^{n}\right) & =0,
\end{aligned}
$$

are true since $V_{1}^{K}$ and $V_{2}^{K}$ are both deterministic functions of $\left(S_{1}^{n}, S_{2}^{n}\right)$. Therefore, it follows that

$$
\begin{aligned}
& I\left(X_{1}^{n} ; X_{2}^{n}\right) \\
\leq & I\left(S_{1}^{n} ; S_{2}^{n}\right)+H\left(V_{2}^{K} \mid S_{1}^{n}\right)+H\left(V_{1}^{K} \mid S_{2}^{n}\right) \\
\leq & I\left(S_{1}^{n} ; S_{2}^{n}\right)+H\left(V_{2}^{K}\right)+H\left(V_{1}^{K}\right) \\
\leq & I\left(S_{1}^{n} ; S_{2}^{n}\right)+2 n C_{0} .
\end{aligned}
$$

Combining (9) with (19) we have

$$
n R \leq n C_{1}+n C_{2}+2 n C_{0}-I\left(X_{1}^{n} ; X_{2}^{n}\right)+n \epsilon .
$$

Finally, we use techniques in [4] to single-letterize the bound in 20 to obtain that

$$
\begin{aligned}
2 R \leq & C_{1}+C_{2}+2 C_{0}+I\left(X_{1}, X_{2} ; Y \mid U\right) \\
& +I\left(X_{1} ; U \mid X_{2}\right)+I\left(X_{2} ; U \mid X_{1}\right)
\end{aligned}
$$

for every auxiliary channel $p\left(u \mid x_{1}, x_{2}, y\right)$. Combining with the cut-set bound, we finished the proof.

Theorem 2. For diamond network with conferencing relays, rate $R$ is achievable if for some pmf $p\left(u, x_{1}, x_{2}, y\right)=p\left(u, x_{1}, x_{2}\right) p\left(y \mid x_{1}, x_{2}\right)$ and $U \in \mathcal{U}$ with $|\mathcal{U}| \leq \min \left\{\left|\mathcal{X}_{1}\right|\left|\mathcal{X}_{2}\right|+2,|\mathcal{Y}|+4\right\}$, it satisfies that

$$
R \leq \min \left\{\begin{array}{l}
C_{0}+C_{2}+I\left(X_{1} ; Y \mid X_{2}, U\right) \\
C_{0}+C_{1}+I\left(X_{2} ; Y \mid X_{1}, U\right) \\
C_{1}+C_{2}-I\left(X_{1} ; X_{2} \mid U\right) \\
I\left(X_{1}, X_{2} ; Y\right) \\
\frac{1}{2}\left(C_{1}+C_{2}+2 C_{0}-I\left(X_{1} ; X_{2} \mid U\right)\right. \\
\left.\quad+I\left(X_{1}, X_{2} ; Y \mid U\right)\right) .
\end{array}\right.
$$

Proof: The bound is achieved using following scheme.

1) Codebook Generation: Generate $2^{n\left(R_{0}+R_{10}+R_{20}\right)}$ sequences $u^{n}\left(w_{0}, w_{10}, w_{20}\right) \quad$ independently, in an i.i.d. fashion according to $\prod_{i=1}^{n} p_{U}\left(u_{i}\right)$. For each sequence $u^{n}\left(w_{0}, w_{10}, w_{20}\right)$, generate $2^{n R_{1}}$ sequences $x_{1}^{n}\left(w_{0}, w_{10}, w_{20}, w_{1}\right)$ independently in an i.i.d. fashion according to $\prod_{i=1}^{n} p_{X_{1} \mid U}\left(x_{1 i} \mid u_{i}\right)$ and likewise for $x_{2}^{n}\left(w_{0}, w_{10}, w_{20}, w_{2}\right)$. For each sequence $u^{n}\left(w_{0}, w_{10}, w_{20}\right)$, pick $2^{n R^{\prime}}$ sequence pairs $\left(x_{1}^{n}\left(w_{0}, w_{10}, w_{20}, w_{1}\right), x_{2}^{n}\left(w_{0}, w_{10}, w_{20}, w_{2}\right)\right)$ that are jointly typical. Index such pairs by $w^{\prime} \in\left[1: 2^{n R^{\prime}}\right]$.

2) Encoding: To transmit message $W=$ $\left(w_{0}, w_{10}, w_{2,0}, w^{\prime}\right)$, the encoder transmits $w_{0}, w_{10}$, and $w_{1}$ through the bit-pipe to relay 1 , and $w_{0}, w_{20}$, and $w_{2}$ through the bit-pipe to relay 2 $\left(x_{1}^{n}\left(w_{0}, w_{10}, w_{20}, w_{1}\right)\right.$ and $x_{2}^{n}\left(w_{0}, w_{10}, w_{20}, w_{2}\right)$ are the $m^{\prime}$ th jointly typical pair for $\left.\left(w_{0}, w_{1,0}, w_{2,0}\right)\right)$, in which $w_{0} \in\left[1: 2^{n R_{0}}\right], w_{i, 0} \in\left[1: 2^{n R_{i, 0}}\right], w_{i} \in\left[1: 2^{n R_{i}}\right]$ for $i=1,2$. Then relay 1 transmits $w_{10}$ to relay 2 through the conferencing bit-pipe, and relay 2 transmits $w_{20}$ to relay 1 through the bit-pipe as well. Relay 1 and relay 2 send $x_{1}^{n}\left(w_{0}, w_{10}, w_{20}, w_{1}\right)$ and $x_{2}^{n}\left(w_{0}, w_{10}, w_{20}, w_{2}\right)$ through the MAC, respectively. 
3) Decoding: Define $w_{c}=\left(w_{0}, w_{10}, w_{20}\right)$. Upon receiving $y^{n}$, the receiver looks for $\left(\hat{w}_{c}, \hat{w}^{\prime}\right)$ for which:

$\left(u^{n}\left(\hat{w}_{c}\right), x_{1}^{n}\left(\hat{w}_{c}, \hat{w}_{1}\right), x_{2}^{n}\left(\hat{w}_{c}, \hat{w}_{2}\right), y^{n}\right) \in \mathcal{T}_{\epsilon}^{n}$, where $\mathcal{T}_{\epsilon}^{n}$ denotes the jointly typical set of $\left(U, X_{1}, X_{2}\right)$.

4) Error Analysis: The error analysis is an extension to [4] with the added common message components from the relay conferencing links, and are omitted here due to limited space. Cardinality bounds on $U$ are derived using standard techniques [6, Appendix C].

\section{CAse Studies}

\section{A. Symmetric Gaussian Diamond Channel}

In this section, we consider a special case of the diamond network investigated, with $C_{1}=C_{2}=C$ and Gaussian MAC. The output of the Gaussian MAC is

$$
Y=X_{1}+X_{2}+Z \text {, }
$$

where $Z \sim \mathcal{N}(0,1)$. Both $X_{1}$ and $X_{2}$ have average power constraint $\frac{1}{n} \sum_{i=1}^{n} E\left[X_{j, i}^{2}\right] \leq P$ for $j=1,2$.

Now we apply Theorem 1 to obtain an upper bound for this setup with techniques similar to [4]. After simplification, we obtain upper bound as:

$$
C^{+}=\max \left\{\max _{\rho \leq \rho *} S_{1}(\rho), \max _{\rho * \leq \rho \leq 1} S_{2}(\rho)\right\} .
$$

where

$$
\begin{aligned}
& S_{1}(\rho)=\min \left\{\begin{array}{l}
2 C \\
\frac{1}{2} \log (1+2(1+\rho) P) \\
C+C_{0}+\frac{1}{2} \log \left(1+\left(1-\rho^{2}\right) P\right) \\
C+C_{0}+\frac{1}{4} \log (1+2(1+\rho) P) \\
-\frac{1}{2} \log \frac{1}{1-\rho^{2}}
\end{array}\right. \\
& S_{2}(\rho)=\min \left\{\begin{array}{l}
2 C \\
\frac{1}{2} \log (1+2(1+\rho) P) \\
C+C_{0}+\frac{1}{2} \log \left(1+\left(1-\rho^{2}\right) P\right)
\end{array}\right. \\
& \rho *=\sqrt{1+\frac{1}{4 P^{2}}-\frac{1}{2 P} .}
\end{aligned}
$$

Lower bound is evaluated from (22) by choosing $\left(U, X_{1}, X_{2}\right)$ to be jointly Gaussian, i.e. $\left(U, X_{1}, X_{2}\right) \sim$ $\mathcal{N}\left(0, \mathbf{K}_{U X_{1} X_{2}}\right)$ and optimizing over $\mathbf{K}_{U X_{1} X_{2}}$.

For the case $P=1$, the results are shown in Fig. 2(a) and Fig. 2(b) for the upper and lower bounds, respectively. The gap between the upper and lower bounds can be seen more clearly from Fig. 2(c) with $C_{0}=0,0.3$ and 0.6 .

\section{B. Minimium $C_{0}$ Required for Full Cooperation for Symmetric Gaussian MAC}

The minimum conferencing link capacity $C_{0}$ required such that full-cooperation capacity $\frac{1}{2} \log (1+4 P)$ can be achieved for the Gaussian symmetric diamond network is summarized in Theorem 3. Note that the regime of interest is $\frac{1}{4} \log (1+4 P) \leq C<\frac{1}{2} \log (1+4 P)$ since for $C<\frac{1}{4} \log (1+4 P)$ the sum capacity of the sourceto-relays bit-pipe links $2 C$ becomes the bottleneck and for $C \geq \frac{1}{2} \log (1+4 P)$, minimum $C_{0}$ required is zero because repetitive message can be sent via both links to obtain full cooperation.

Theorem 3. Given the capacity of the source-to-relay bit-pipe links $C \in\left[\frac{1}{4} \log (1+4 P), \frac{1}{2} \log (1+4 P)\right]$, the cut-set bound $R \leq \frac{1}{2} \log (1+4 P)$ can be achieved if and only if $C_{0} \geq \frac{1}{2} \log (1+4 P)-C$.

Proof: The achievability part is obvious. For the converse part, note that for (25), in the case of $\frac{1}{4} \log (1+$ $4 P) \leq C<\frac{1}{2} \log (1+4 P)$, the constraint $2 C$ for both $S_{1}(\rho)$ and $S_{2}(\rho)$, respectively, is inactive.

Now, in this regime, observe that

$$
\begin{aligned}
\max _{\rho \leq \rho *} S_{1}(\rho) & \leq \max _{\rho \leq \rho *} \frac{1}{2} \log (1+2(1+\rho) P) \\
& <\frac{1}{2} \log (1+4 P),
\end{aligned}
$$

thus we have $C^{+}=\frac{1}{2} \log (1+4 P)$ if and only if $\max _{\rho * \leq \rho \leq 1} S_{2}(\rho)=\frac{1}{2} \log (1+4 P)$. Since for $\rho *<\rho<1$ we have

$$
S_{2}(\rho) \leq \frac{1}{2} \log (1+2(1+\rho) P)<\frac{1}{2} \log (1+4 P),
$$

$\max _{\rho * \leq \rho \leq 1} S_{2}(\rho)=\frac{1}{2} \log (1+4 P)$ is satisfied if and only if $S_{2}(1)=\frac{1}{2} \log (1+4 P)$, which implies

$$
C+C_{0} \geq \frac{1}{2} \log (1+4 P)
$$

which completes the converse proof.

\section{Diamond Network with Binary Adder MAC}

The diamond network under consideration here has the MAC portion as a binary adder channel (BAC). The output of BAC is $Y=X_{1}+X_{2}$, where the input alphabet is $\mathcal{X}=\{0,1\}$ and the output alphabet is $\mathcal{Y}=\{0,1,2\}$. We let $C_{1}=C_{2}=C$. For the achievability, we utilise (22) to obtain:

$$
R \leq \min \left\{\begin{array}{l}
H\left(X_{1} \mid X_{2}, U\right)+C+C_{0} \\
H\left(X_{2} \mid X_{1}, U\right)+C+C_{0} \\
2 C-I\left(X_{1} ; X_{2} \mid U\right) \\
H(Y) \\
\frac{1}{2}\left(H(Y \mid U)+2 C+2 C_{0}-I\left(X_{1} ; X_{2} \mid U\right)\right)
\end{array}\right.
$$

Figures on bounds are omitted due to limited space.

\section{Minimium $C_{0}$ Required for Full Cooperation for Binary Adder MAC}

The result in Theorem 3 does not hold in general. As a counter-example, consider the diamond channel with binary adder MAC. The minimum $C_{0}$ can only be obtained numerically here and the result shows that there 

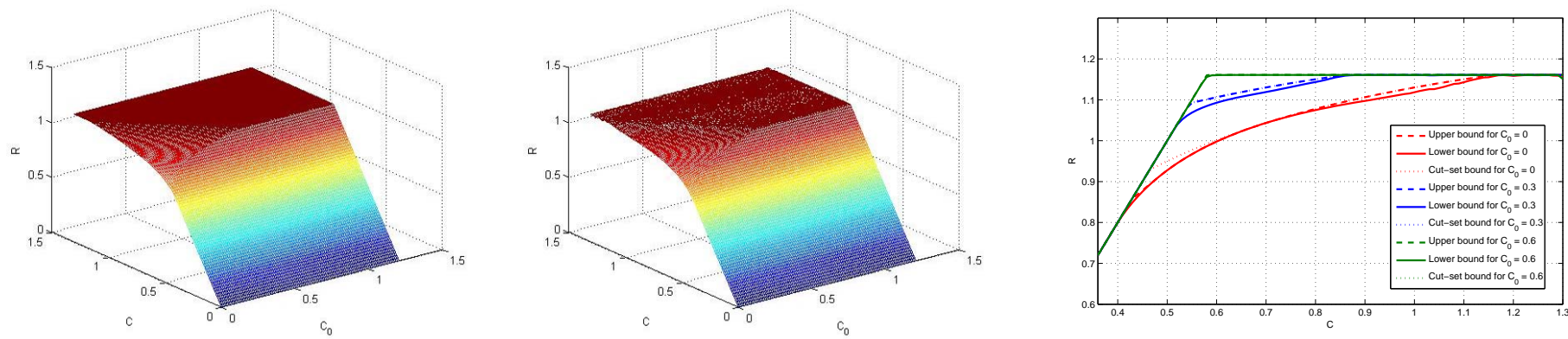

(a) Upper bound for the rate of Gaussian sym- (b) Lower bound for the rate of Gaussian (c) Bounds for the rate of Gaussian symmetric diamond metric diamond network with conferencing symmetric diamond network with conferenc- network with relay conference as a function of $C$ for relays when $P=1$. ing relays when $P=1$. $C_{0}=0,0.3$ and 0.6 when $P=1$.

Fig. 2. Results on symmetric Gaussian diamond channel.

is a gap between the upper bound and lower bound about the minimum $C_{0}$ required to achieve cut-set bound, as seen from Fig. 3

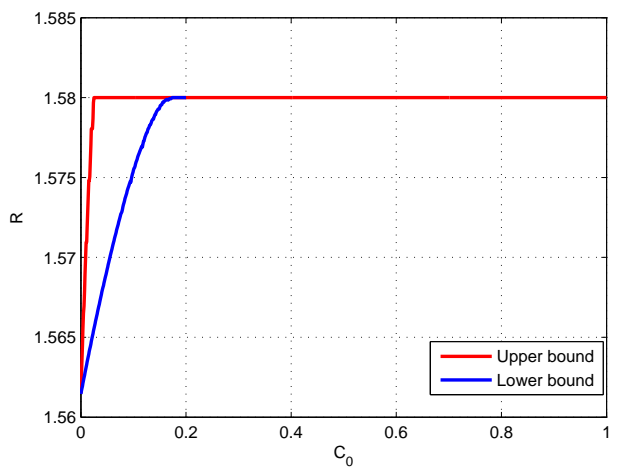

Fig. 3. Upper and lower bound for binary adder diamond channel with conference with $C=0.79$.

Fig. 3 shows that the cut-set bound 1.58 for $C=0.79$ is achievable if $C_{0} \geq 0.174$ and only if $C_{0} \geq 0.025$. It can be observed that unlike the Gaussian case, the minimum $C_{0}$ needed is much less than the difference between the cut-set bound 1.58 and $C=0.79$. To briefly explain such phenomenon, first denote the optimal distribution in the cut-set bound $R_{C S}=\max _{p\left(x_{1}, x_{2}\right)} I\left(X_{1}, X_{2} ; Y\right)$ as $p\left(x_{1}, x_{2}\right)^{*}$. For the lower bound given in (22), to achieve this $R_{C S}$ for the case $C=\frac{1}{2} R_{C S}$ as an example, we need (i) $p\left(u, x_{1}, x_{2}\right)=p(u) p\left(x_{1} \mid u\right) p\left(x_{2} \mid u\right)$ (to make $I\left(X_{1} ; X_{2} \mid U\right)=0$ ), (ii) $\sum_{u \in \mathcal{U}} p\left(u, x_{1}, x_{2}\right)=$ $p\left(x_{1}, x_{2}\right)^{*}$ and $C+C_{0}+I\left(X_{1} ; Y \mid X_{2}, U\right) \geq R_{C S}$, $C+C_{0}+I\left(X_{2} ; Y \mid X_{1}, U\right) \geq R_{C S}$, as well as $C+$ $C_{0}+\frac{1}{2} I\left(X_{1}, X_{2} ; Y \mid U\right) \geq R_{C S}$, which yields different lower bounds for $C_{0}$ for different models. For the Gaussian case, $p\left(u, x_{1}, x_{2}\right)$ satisfying $(i)(i i)$ will always make $I\left(X_{1}, X_{2} ; Y \mid U\right)=0$ (hence $I\left(X_{1} ; Y \mid X_{2}, U\right)=0$ ) because for the optimal $p\left(x_{1}, x_{2}\right)^{*}$, we have $X_{1}=X_{2}$, to form Markov chain $X_{1} \leftrightarrow U \leftrightarrow X_{2}, U$ has to be picked as a deterministic function of $X_{1}$ (or $\left.X_{2}\right)$. However, for the binary adder case, there exists $p\left(u, x_{1}, x_{2}\right)$ satisfying $(i)(i i)$ such that $I\left(X_{1} ; Y \mid X_{2}, U\right)>$ $0, I\left(X_{2} ; Y \mid X_{1}, U\right)>0$ and $\frac{1}{2} I\left(X_{1}, X_{2} ; Y \mid U\right)>0$. For example, with $p(u)=\left[\frac{1}{2}, \frac{1}{2}\right], p\left(x_{1} \mid u\right)=p\left(x_{2} \mid u\right)=$ $[\alpha, 1-\alpha ; \beta, 1-\beta]$ with $\alpha=\frac{1}{2}-\frac{\sqrt{3}}{6}, \beta=\frac{1}{2}+\frac{\sqrt{3}}{6}$, we have $\sum_{u \in \mathcal{U}} p\left(u, x_{1}, x_{2}\right)=\left[\frac{1}{3}, \frac{1}{6} ; \frac{1}{6}, \frac{1}{3}\right]$ and $I\left(X_{1} ; Y \mid X_{2}, U\right)=$ $I\left(X_{2} ; Y \mid X_{1}, U\right)=0.7440, \frac{1}{2} I\left(X_{1}, X_{2} ; Y \mid U\right)=0.5774$, hence when $C_{0} \geq 0.2152, R_{C S}$ of 1.5850 can be achieved given $C=0.7925=\frac{1}{2} R_{C S}$.

\section{CONCLUSION}

In this letter, effects of relay conferencing on a class of diamond networks are examined. A lower bound to the capacity for this network and an upper bound that is tighter than the cut-set bound are presented. The bounds applied to specific MAC cases reveal a special property of the Gaussian channel in that there is no clever manipulation of conferencing links to achieve full MAC cooperation with individual source-to-relay messages. However, such is not the case in general.

\section{REFERENCES}

[1] B. E. Schein, "Distributed coordination in network information theory," Ph.D. dissertation, Massachusetts Institute of Technology, 2001.

[2] D. Traskov and G. Kramer, "Reliable communication in networks with multi-access interference," in Proc. Info. Theory Workshop, Sept 2007, pp. 343-348.

[3] W. Kang and N. Liu, "The gaussian multiple access diamond channel," in Proc. Int. Symp. Inf. Theory, July 2011, pp. 1499 1503.

[4] S. Shirin and G. Kramer, "Capacity bounds for a class of diamond networks," in Proc. Int. Symp. Inf. Theory, June 2014, pp. 1196-1200.

[5] F. Willems, "The discrete memoryless multiple access channel with partially cooperating encoders (corresp.)," IEEE Trans. Inf. Theory, vol. 29, no. 3, pp. 441-445, May 1983.

[6] A. E. Gamal and Y.-H. Kim, Network Information Theory. Cambridge University Press, 2011. 